\title{
Model Predictive Control of Precision Air Conditioning System with Secondary Condenser
}

\author{
Iput Kasiyanto $^{1}$, Aries Subiantoro ${ }^{1}$ \\ iput.kasiyanto@ui.aci.id, biantoro@ee.ui.ac.id \\ Universitas Indonesia ${ }^{1}$
}

\begin{abstract}
Due to high specification of ASHRAE requirements, a data center (DC) must have sophisticated cooling system to maintain its environment conditions. Its high dimensional coupling system dynamics makes controller design very challenging and complicated. A non-linear system model of a precision air conditioning (PAC) system with secondary condenser has been previously developed and then linearized at steady state operating point. Based on the model, a model predictive control (MPC) based controller is designed to deal with coupling state variables(i.e: temperature and relative humidity). The controller also has ability to improve energy efficiency of the PAC system. The performance of the proposed controller is validated through simulation. The results showed the effectiveness of MPC based controller against the defined constraints.
\end{abstract}

Keywords: Energy efficiency; Model predictive control; Precision air conditioning.

\section{Introduction}

For the last two decades, as the strengthening people awareness on sustainable energy future, energy saving strategies have become top priority in energy policies in many developed countries all over the world, especially with the significant increase of energy consumption in buildings[1]. For example, in 2004 building consumption in the EU was 37\% of final energy[2], bigger than industry (28\%) and transport (32\%). Meanwhile, the USA's building energy consumption accounted for $41 \%$ of primary energy consumption in 2010[3]. Mostly, the categories of building services and heating, ventilation, and air conditioning (HVAC) systems in developed countries constitute the major sources of energy use in buildings[1],[2]. Thus, the research and development and their subsequent implementations of effective control strategies for HVAC systems become primarily important and urgent.

Precision air conditioning (PAC) systems, which belong to HVAC applications, are widely used in data centers(DCs) to maintain the temperature and relative humidity of DCs in an appropriate condition as specified by ASHRAE requirements[4]. PAC system typically eliminates heat produced by the DC equipments using vapour-compression process cycle. Early studies on HVAC control focusing on multiple loops of system-input-system-output (SISO) PIbased controllers have proved that the control strategies only benefited low gains. In addition, its tedious and (sometimes) inaccurate tuning of classical PI-based HVAC controllers contributed to poor performance[5],[6]. Also, SISO controllers are unable to handle cross-coupling nature in an HVAC system. Therefore, multivariable control strategies become interesting options. Among many multivariable control strategies, model predictive control (MPC) approach has several advantages[7], which include: utilize of a mathematical model for antici- 
patory control actions instead of corrective control, incorporation of a disturbance model for disturbance rejection, ability to deal with constraints and uncertainties, ability to handle slowdynamics processes with time delays, use of a cost function for satisfying multiple objectives, and utilize of advanced optimization methods for computing of control vectors. The basic concept of MPC approach is to use a system model to predict the future states of the system using the curent and past measurements. The controller, then, generates a control vector that minimizes a certain cost function over the prediction horizon in the presence of disturbances and constraints. Many MPC applications in HVAC systems have been studied and implemented, for instances: in controlling VAV zone temperature and damper position[8], for the charging and discharging control of an ice storage system[9], in reducing energy consumption of heat pump of a solar house[10], in controlling the optimal temperature of a commercial building[11], in reducing the operating and maintenance cost in a district heating power plant [12], for maintaining the indoor thermal comfort in IoT Smart Space[13], and many others.

In order to overcome the drawbacks of convensional-method-based controller as mentioned before(i.e. : being not able to handle coupled variables in MIMO system, not able to incorporate constraints in control design, and the irksomely need of retuning controller parameters), the new method of controller was proposed. This paper presents MPC controller design based on the previously developed model by Subiantoro et al[14]. The PAC system model is derived using psychrometric data and underlying physics laws, i.e.: the conservation of mass and energy balance principles. The previous work was only the dynamic model of PAC system with new additional component, namely a secondary condenser to improve regulation of relative humidity. But the controller for the system was not designed yet. Thus, based on the model, a model predictive control (MPC) is designed to deal with coupling state variables(i.e: temperature and relative humidity) and to improve energy efficiency of the PAC system. The performance of the proposed controller is validated through simulation.

\section{Methods}

This section consists of two sub sections. The first part describes briefly the PAC model used as controlled plant. Then, the controller design which applied to the plant is explained in the second part.

\subsection{PAC System Model}

As mentioned before, the model used in the controller design is developed by Subiantoro et al[14]. The schematic representation of the model is shown in Figure 1. The system consists of basic HVAC components, namely: a compressor, heat exchanger components (an evaporator, two condensers and a caviler pipe), two fans and regulation valves (an electronic valve and a check valve). It is mainly composed of two parts, refrigerant-side and an air-side. The secondary condenser is placed at PAC outlet to work as an air heating coil. The system uses refrigerant $\mathrm{R} 134 \mathrm{a}$ as its working fluid, with a total charge of $0.5 \mathrm{~kg}$.

The dynamic model is derived using energy and mass conservation laws for components composing the PAC system, which includes: compressor model, evaporator model, secondary condenser, and cabinet model. For detail derivation, one can refer to[14]. 


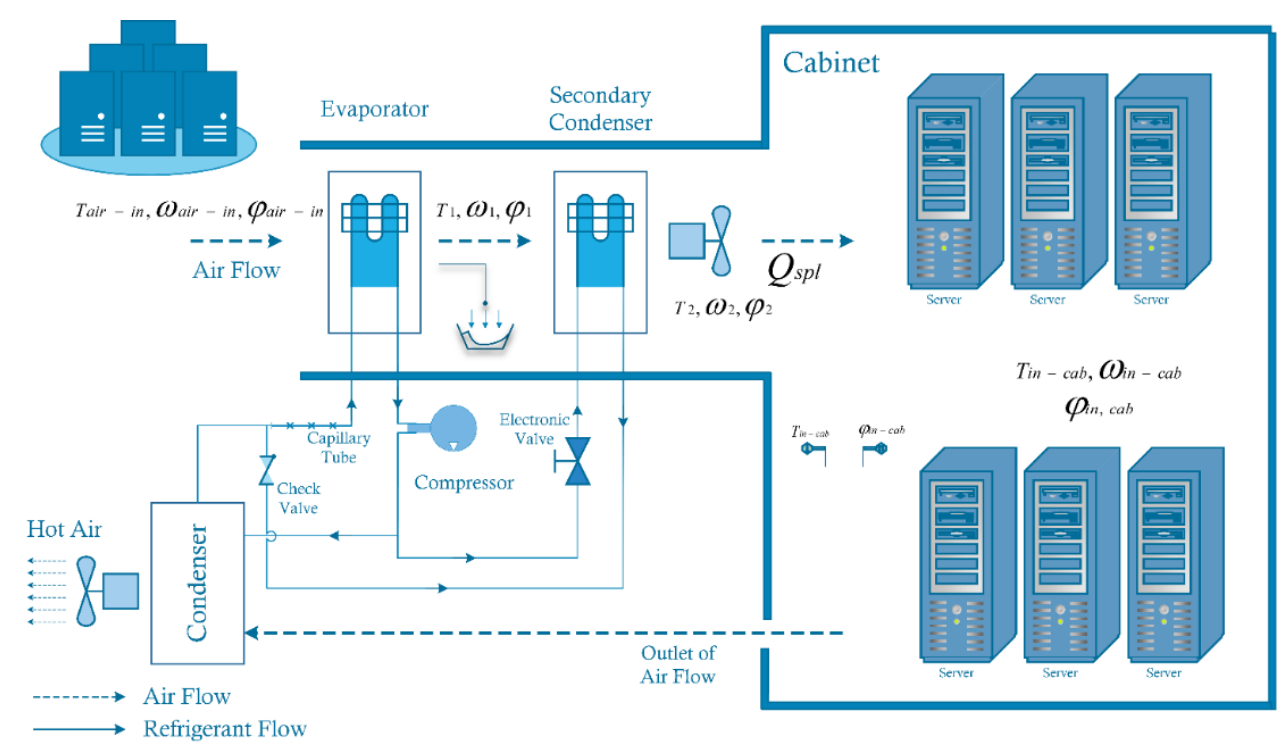

Fig. 1. Schematic Representation of the PAC System[14].

The developed model is nonlinear. The model can be represented in state-space form as

$$
\dot{\boldsymbol{x}}=\boldsymbol{H}^{-\mathbf{1}} \boldsymbol{f}_{\mathbf{1}}(\boldsymbol{x}, \boldsymbol{u}, t)+\boldsymbol{H}^{-\mathbf{1}} \boldsymbol{f}_{\mathbf{2}}(\boldsymbol{n}, t)
$$

where $\boldsymbol{x}$ is state variables vector defined as $\boldsymbol{x}=\left[T_{c a b}, \omega_{c a b}, T_{1}, T_{1}^{\prime}, T_{2}, T_{w e}, T_{w c 2}, \omega_{1}\right]^{T}, \boldsymbol{u}$ is input variables vector defined as $\boldsymbol{u}=[f, s]^{T}$, and $\boldsymbol{n}$ is disturbance variables vector defined as $\mathbf{n}=\left[T_{\text {air-in }}, \omega_{\text {air-in }}, Q_{\text {load }}, M\right]^{T} . \boldsymbol{H}^{-\mathbf{1}}$ is defined in Appendix A of the preliminary work[14].

In order to be convenient for designing multivariable control, the nonlinear model should be linearized about its operating point as described in Table 1.

Table 1. The Operating Point of the PAC System.

\begin{tabular}{cccc}
\hline Variable & Numerical value & Variable & Numerical value \\
\hline$T_{c a b}$ & $25.2051^{\circ} \mathrm{C}$ & $T_{w e}$ & $3.0266^{\circ} \mathrm{C}$ \\
$T_{1}$ & $22.4299^{\circ} \mathrm{C}$ & $T_{1}^{\prime}$ & $23.0859{ }^{\circ} \mathrm{C}$ \\
$T_{2}$ & $23.4183^{\circ} \mathrm{C}$ & $T_{w c 2}$ & $24.8727^{\circ} \mathrm{C}$ \\
$\omega_{1}$ & $0.0102 \mathrm{~kg} / \mathrm{kg}$ & $\omega_{c a b}$ & $0.0104 \mathrm{~kg} / \mathrm{kg}$ \\
$s$ & $60 \mathrm{rps}$ & $f$ & $0.04722 \mathrm{~m}^{3} / \mathrm{s}$ \\
\hline
\end{tabular}


Applying Taylor series method to equation (1) and calculate it about its operating point, the linearized dynamic model can be written as state-space representation in the following compact form

$$
\dot{x}=A_{o p} x+B_{o p} u+V_{o p} n
$$

where $\boldsymbol{A}_{\boldsymbol{o p}}, \boldsymbol{B}_{\boldsymbol{o p}}$, and $\boldsymbol{V}_{\boldsymbol{o p}}$ defined in [14].

Meanwhile, the output of the PAC system is calculated using linear regression method considering linear relationship between the relative humidity of cabinet and the specific humidity of cabinet. The output of the system can be written as follows

$$
y=C_{o p} x+d_{o p}
$$

where $\mathbf{y}$ is output variables vector defined as $\boldsymbol{y}=\left[T_{c a b}, \phi_{c a b}\right]^{T}$, with $\boldsymbol{C}_{\boldsymbol{o p}}$ and $\boldsymbol{d}_{\boldsymbol{o p}}$ defined in[14].

\subsection{MPC Controller Design}

As digital controller, the MPC controller performs all estimation and optimization computations in discrete-time domain. Therefore, the model described in equation (2) and (3) have to be discretized in the following form

$$
\left\{\begin{array}{c}
\boldsymbol{x}_{k+1}=\boldsymbol{A}_{\boldsymbol{d}} \boldsymbol{x}_{k}+\boldsymbol{B}_{\boldsymbol{d}} \boldsymbol{u}_{k}+\boldsymbol{V}_{\boldsymbol{d}} \boldsymbol{n}_{k} \\
\boldsymbol{y}_{k}=\boldsymbol{C}_{\boldsymbol{d}} \boldsymbol{x}_{k}+\boldsymbol{d}_{k}
\end{array}\right.
$$

where $\boldsymbol{A}_{\boldsymbol{d}}, \boldsymbol{B}_{\boldsymbol{d}}, \boldsymbol{C}_{\boldsymbol{d}}$ and $\boldsymbol{V}_{\boldsymbol{d}}$ respectively are discrete form of matrices $\boldsymbol{A}_{\boldsymbol{o p}}, \boldsymbol{B}_{\boldsymbol{o p}}, \boldsymbol{C}_{\boldsymbol{o p}}$ and $\boldsymbol{V}_{\boldsymbol{o p}}$. Meanwhile, $\boldsymbol{x}_{k}, \boldsymbol{u}_{k}, \boldsymbol{n}_{k}$ and $\boldsymbol{y}_{k}$ respectively are discrete form of vector $\boldsymbol{x}, \boldsymbol{u}, \boldsymbol{n}$ and $\boldsymbol{y}$ defined as in the previous subsection.

The main idea of MPC, as indicated by its name, is to predict the future states of the controlled system and subsequently to generate a control vector that minimizes a certain cost function over finite prediction horizon in the existence of disturbances and constraints. For each sampling period only the first computed control element is applied to the system input, and the remainder is eliminated. The process is repeated again in the next instant. Thus, the described algorithm is also called receding horizon control. For a current system state $\boldsymbol{x}_{k}$, the control input is determined by the solution of

$$
\boldsymbol{u}_{k}^{*}=\left[\begin{array}{llll}
1 & 0 & \cdots & 0
\end{array}\right]\left(\arg \min _{\left[\boldsymbol{u}_{k}, \cdots, \boldsymbol{u}_{k+n_{C}}\right]^{T}} J\left(\boldsymbol{x}_{k}, \boldsymbol{u}_{k-1}\right)\right)
$$

subject to system, control and optimization constraints[15]. $\boldsymbol{u}_{k}^{*}$ is the first control input from the calculated set of optimal control inputs $\left\{\boldsymbol{u}_{k}^{*}, \cdots, \boldsymbol{u}_{k+M}^{*}\right\}$ for a control horizon of length $n_{C}$. The MPC uses the cost function $J_{k}$ to penalize (a) deviations of the predicted outputs from a reference (setpoint) trajectory and (b) the smoothness of manipulated variable. 
The cost function can be expressed in a discrete-time linear quadratic functional as follows

$$
J_{k}=\overbrace{\sum_{i=0}^{n_{P}}\left\|\boldsymbol{r}_{k+i}-\widehat{\boldsymbol{y}}_{k+i \mid k}\right\|_{W^{Y}}^{2}}^{a}+\overbrace{\sum_{i=0}^{n_{C^{-}}}\left\|\Delta \boldsymbol{u}_{k+i}\right\|_{\boldsymbol{W}^{U}}^{2}}^{b}
$$

where $k$ is the current time index, $n_{P}$ is the prediction horizon, $n_{C}$ is the control horizon, $\boldsymbol{r}_{k+i}$ is the reference vector at step $k, \widehat{\boldsymbol{y}}_{k+i \mid k}$ is the predicted output vector at step $k+i, \Delta \boldsymbol{u}_{k+i}=$ $\boldsymbol{u}_{k+i}-\boldsymbol{u}_{k+i-1}$ is the variation of manipulated variable [11]. $\|.\|_{\text {(.) }}^{2}$ indicates a square of an Euclidean vector norm weighted over a specified matrix $\left(\|\boldsymbol{x}\|_{W}^{2}=\boldsymbol{x}^{T} \boldsymbol{W} \boldsymbol{x}\right),\left(\boldsymbol{W}^{\boldsymbol{Y}}\right)^{T} \geqslant \mathbf{0}$ and $\left(\boldsymbol{W}^{U}\right)^{T} \geqslant \mathbf{0}$.

The $n_{P}$-step output prediction used in $J_{k}$ is determined in the following equation:

$$
\begin{aligned}
& \overbrace{\left[\begin{array}{c}
\hat{y}_{k+1 \mid k} \\
\hat{y}_{k+2 \mid k} \\
\vdots \\
\hat{y}_{k+n_{P} \mid k}
\end{array}\right]}^{\hat{Y}}=\overbrace{\left[\begin{array}{c}
C A \\
C A^{2} \\
\vdots \\
C A^{n_{P}}
\end{array}\right] \widehat{\boldsymbol{x}}_{k \mid k}+\left[\begin{array}{c}
C B \\
C A B+C B \\
\vdots \\
\sum_{i=1}^{n_{P}} C A^{i-1} B
\end{array}\right] \boldsymbol{u}_{k-1}}^{\vartheta}+ \\
& +\underbrace{\left[\begin{array}{cccc}
C B & \cdots & 0 & 0 \\
C A B & C B & & \vdots \\
\vdots & & \ddots & 0 \\
\sum_{i=1}^{n_{P}} C A^{i-1} B & \cdots & & \sum_{i=1}^{n_{P}-n_{C}+1} C A^{i-1} E
\end{array}\right]}_{S_{\vartheta}} \underbrace{\left[\begin{array}{c}
\Delta u_{k} \\
\Delta u_{k+1} \\
\vdots \\
\Delta u_{k+n_{C}-1}
\end{array}\right]}_{\Delta u}
\end{aligned}
$$

The quadratic programing problem of MPC can defined as

$$
\min _{\Delta u} J_{k}=\frac{1}{2} \Delta \boldsymbol{u}^{T} \boldsymbol{\psi} \Delta \boldsymbol{u}+\boldsymbol{\vartheta}^{T} \Delta \boldsymbol{u}
$$

subject to:

$$
\boldsymbol{y}_{\min } \leq \boldsymbol{y}_{k} \leq \boldsymbol{y}_{\max } ; \boldsymbol{u}_{\min } \leq \boldsymbol{u}_{k} \leq \boldsymbol{u}_{\max }
$$

where:

$$
\begin{gathered}
\boldsymbol{\psi}=\boldsymbol{S}_{\boldsymbol{\vartheta}}^{T} \boldsymbol{W}^{Y} \boldsymbol{S}_{\boldsymbol{\vartheta}}+\boldsymbol{W}^{U} \\
\boldsymbol{\vartheta}=-\boldsymbol{S}_{\vartheta}^{T} \boldsymbol{W}^{Y} \boldsymbol{\varepsilon} \\
\boldsymbol{\varepsilon}=\boldsymbol{r}-\boldsymbol{\vartheta} \\
\boldsymbol{r}=\left[\begin{array}{llll}
r_{k+1} & r_{k+2} & \cdots & r_{k+n_{P}}
\end{array}\right] ; \boldsymbol{W}^{Y}=\left[\begin{array}{ccc}
w^{y} & \cdots & 0 \\
\vdots & \ddots & \vdots \\
0 & \cdots & w^{y}
\end{array}\right] ; \boldsymbol{W}^{U}=\left[\begin{array}{ccc}
w^{u} & \cdots & 0 \\
\vdots & \ddots & \vdots \\
0 & \cdots & w^{u}
\end{array}\right]
\end{gathered}
$$


The control process of MPC is schematically described in Figure 2. It consists of two main block, namely: MPC block and plant block. The MPC block calculates the optimal control $\boldsymbol{u}_{k}^{*}$ and uses it to control the plant so that it can follow the setpoint trajectory and fulfil the constraints and cost function criteria. Meanwhile, the plant block is the dynamics model for the controlled process as derived in [14]. It contains system parameters and typically includes uncertainties, both model uncertainty and disturbances.

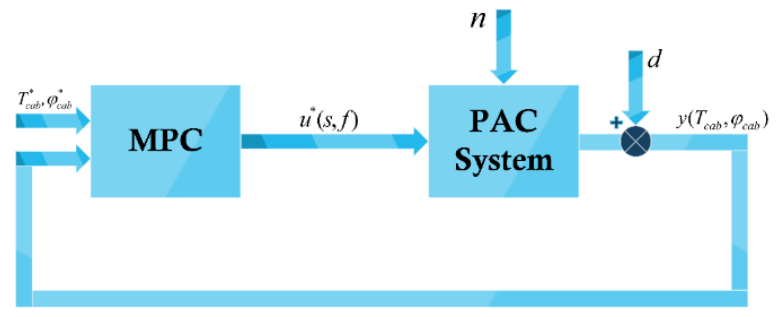

Fig. 2. Schematic Control Diagram of MPC for the PAC System.

\section{Results and Discussions}

For the simulation, the following parameters have been set: prediction horizon $n_{P}=200 \mathrm{~s}$, control horizon $n_{C}=25 \mathrm{~s}$, sampling time $T_{s}=5 \mathrm{~s}$. Meanwhile the constraints for fan and compressor are defined as follows

$$
\begin{gathered}
0.01416 \leq f \leq 0.04722 \\
20 \leq s \leq 73.33
\end{gathered}
$$

The simulation was conducted using well-known general purpose software, Matlab® 2018b. Simulation of MPC controller gave satisfying result as depicted in Figure 3. The contolled variables, i.e: temperature and RH, can follow the setpoint trajectory smoothly. Even there is small overshoot when the setpoint changes, the output variables relatively well decoupled using the controller. The choosing of horizons, both prediction and control horizon, are very crucial in order to get the expected result. Due to the slow dynamics of the system, value of $n_{P}=200 \mathrm{~s}$ and $n_{c}=25 \mathrm{~s}$ for duration 7000s are reasonable [7].

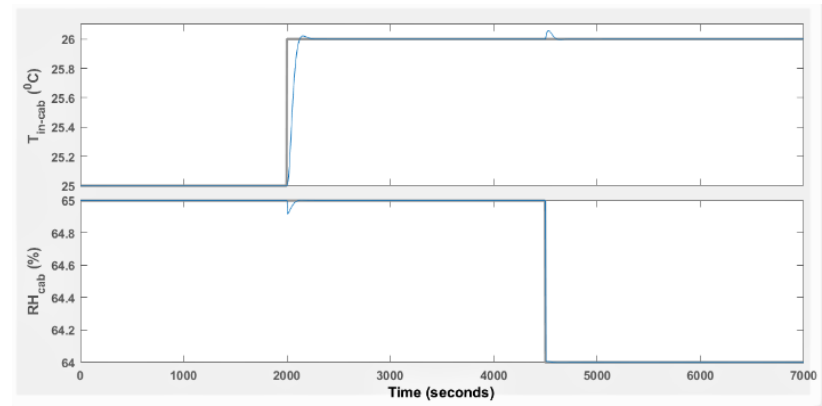

Fig. 3. Output responses of PAC System using MPC. 
When setpoint of $T_{i n-c a b}$ is changed from $25^{\circ} \mathrm{C}$ to $26^{\circ} \mathrm{C}$ in $t=2000 \mathrm{~s}$ while maintaining setpoint $R H_{c a b}$ constant, it can be seen that actual value $R H_{c a b}$ changes as well, though very small amount. In a similar way, the change of setpoint $R H_{c a b}$ from $65 \%$ to $64 \%$ in $t=4500 \mathrm{~s}$ while maintaining setpoint $T_{i n-c a b}$ constant still effects in actual value $T_{i n-c a b}$. The both cases show that there is interaction between the two variables $\left(T_{i n-c a b}\right.$ and $\left.R H_{c a b}\right)$ or in another term, they are coupled each other. However, in both cases, the amount of change responses are very small. Thus, it proved that MPC controller performs very well in decoupling the multivariable system.

Besides the ability of decoupling the MIMO system, there is another advantage of MPC which makes it become preference among process engineers in controlling multivariable systems. It is the ability to handle constraints in a fashionable way. From Figure 4 it can be seen that the values of fan speed $(f)$ and compressor speed $(s)$ are limited in particular range as defined in constraints, i.e.: between $0.01416 \mathrm{~m}^{3} / \mathrm{s}$ and $0.04722 \mathrm{~m}^{3} / \mathrm{s}$ for $f$, while for $s$ between 20 rps and 73.33 rps. In $t=2000 s$, the fan and compressor speed decrease to make system follow setpoint $T_{i n-c a b}$ change. The same way happens when setpoint $R H_{c a b}$ is changed in $t=4500 \mathrm{~s}$. The control inputs change as well, but again still in their range limits. The fan speed is around $0.0142 \mathrm{~m}^{3} / \mathrm{s}$ while the compressor speed is about $25 \mathrm{rps}$.

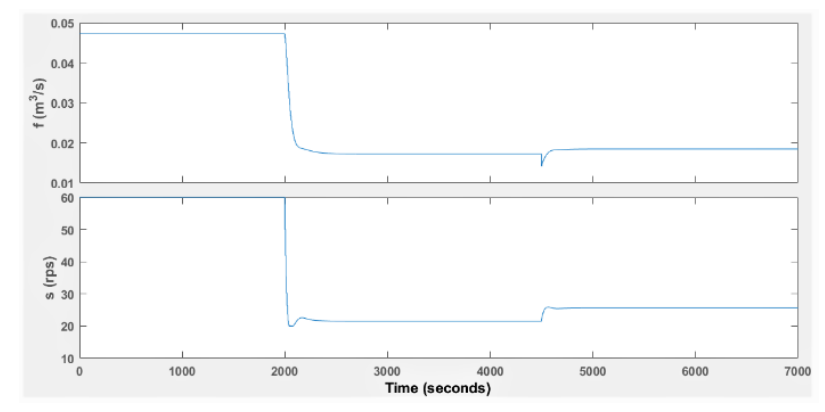

Fig. 4. The value of control input of PAC System using MPC.

As illustrated in Figure 4, the optimization algorithm inside MPC computed the optimal control law such that the value of control inputs will not outside the defined range while in the same time it also satisfies the setpoint conditions.

\section{Conclusion}

An MPC based controller has been designed and validated through simulation. Its performance to control cabinet temperature and relative humidity of PAC system is very well. The controller can handle the cross-coupling variables with satisfying result. It can be seen from the responses in both cases (changing one variable and maintaining the another constant), i.e.: the amount of overshoot responses are very small. Its superiority, particulary in dealing with constraints and cost function, is demonstrated by simulation. Despite the changing of setpoint $T_{i n-c a b}$ and $R H_{c a b}$, the control inputs ( $f$ and $s$ ) still change in their range limits as defined in constraints. 
Acknowledgments. This research is funded by Research Grant of Beasiswa Saintek Kementerian Riset, Teknologi, dan Pendidikan Tinggi.

\section{Nomenclature}

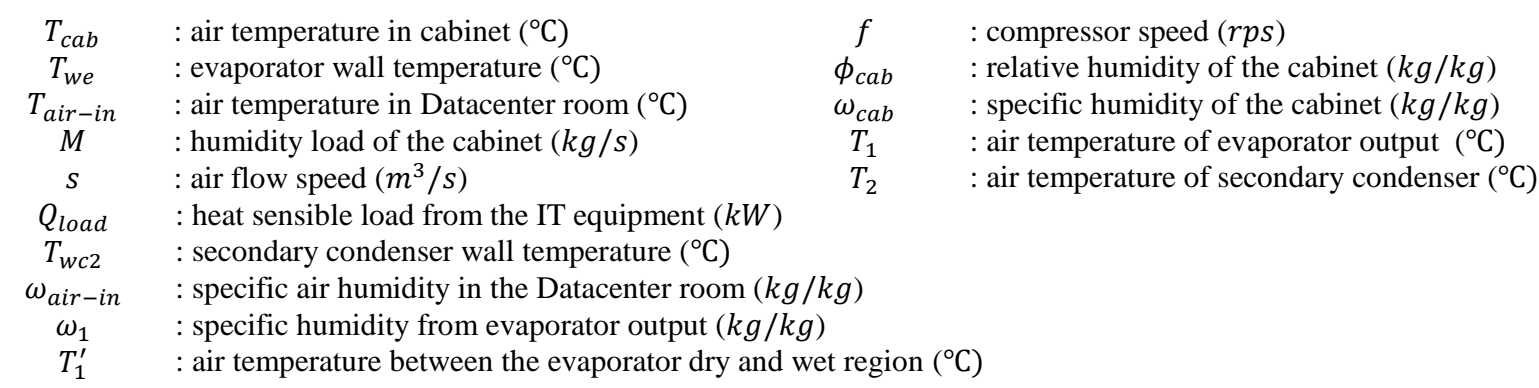

\section{References}

[1] L. Pérez-Lombard, J. Ortiz, and C. Pout.: A review on buildings energy consumption information. Energy Build. (2008)

[2] International Energy Agency. Key World Energy Statistics 2006. (2006)

[3] Annual energy outlook 2012. U.S. Energy Inf. Adm. (2012)

[4] A. Habibi Khalaj and S. K. Halgamuge.: A Review on efficient thermal management of air- and liquid-cooled data centers: From chip to the cooling system. Applied Energy. (2017)

[5] C. G. Nesler and W. F. Stoecker.: Selecting the proportional and integral constants in the direct digital control of discharge air temperature. ASHRAE Trans.. vol. 90. pp. 834-845. (1984)

[6] G. Shavit and S. G. Brandt.: Dynamic performance of a discharge air-temperature system with a P-I controller. in ASHRAE Journal. (1982)

[7] A. Afram and F. Janabi-Sharifi.: Theory and applications of HVAC control systems - A review of model predictive control (MPC). Building and Environment. (2014).

[8] G. Huang.: Model predictive control of VAV zone thermal systems concerning bilinearity and gain nonlinearity. Control Eng. Pract. (2011)

[9] G. P. Henze. R. H. Dodier. and M. Krarti.: Development of a predictive optimal controller for thermal energy storage systems. in ASHRAE Transactions. (1998)

[10] J. A. Candanedo and A. K. Athienitis.: Predictive control of radiant floor heating and solarsource heat pump operation in a solar house. in HVAC and R Research. (2011)

[11] G. Mantovani and L. Ferrarini.: Temperature Control of a Commercial Building with Model Predictive Control Techniques. IEEE Trans. Ind. Electron. (2015)

[12] F. Verrilli et al.: Model Predictive Control-Based Optimal Operations of District Heating System with Thermal Energy Storage and Flexible Loads. IEEE Trans. Autom. Sci. Eng. (2017)

[13] L. Hang and D.-H. Kim.: Enhanced Model-Based Predictive Control System Based on Fuzzy Logic for Maintaining Thermal Comfort in IoT Smart Space. Appl. Sci.. vol. 8. no. 7. p. 1031. Jun. (2018)

[14] A. Subiantoro. Nasruddin. F. Yusivar. M. I. Al-Hamid. and B. Budiardjo.: Modeling of A New Structure of Precision Air Conditioning System Using Secondary Condenser For RH Regulation. MAKARA Technol. Ser.. (2012)

[15] J. Dostál and L. Ferkl.: Model predictive control of climatic chamber with on-off actuators. in IFAC Proceedings Volumes (IFAC-PapersOnline). (2014) 